\title{
CHAVE ILUSTRADA PARA AS ESPÉCIES DE PIPTOCHAETIUM PRESL (GRAMINEAE - STIPEAE) DO BRASIL
}

\author{
Jaime Mujica Sallés' \\ Hilda Maria Longhi-Wagner ${ }^{2}$
}

\begin{abstract}
RESUMO - Até o momento foram encontradas 8 espécies e 6 variedades de Piptochaetium para o Brasil, todas presentes no Rio Grande do Sul, diminuindo este número em direção Norte, sendo que apenas $P$. montevidense alcança a latitude do Trópico de Capricórnio.

É fornecida uma chave ilustradia para a identificação dos táxons, bem como dados sobre sua distribuição geográfica e condições de ocorrência.

Palavras-chave: Gramineae, Pipt ochaetium, Brasil, Sistemática.
\end{abstract}

\begin{abstract}
Eight species and six varieties of Piptochaetium are known for Brazil. All of them occurr in Rio Grande do Sul, but they are less frequent towards the north with only $P$. montevialense reaching the Tropic of Capricorn. An illustrated analytical key for the identification of taxa as well as data on their geographical distribution is provided.
\end{abstract}

Key words: Gramineae, Piptochaet ium, Brazil, Systematics.

\section{Introdução}

O gênero Piptochaetium Presl é exclusivamente americano, apresentando cerca de 30 espécies naturais das regiōes semiáridas e temperadas dos Estados Unidos até a América do Sul. A maior concentração de espécies ocorre no Chile, Argentina, Uruguai e sul dos Brasil.

A descrição do gênero foi feita pcrr Presl em 1830. No trabalho anterior, de Nees (1829), o mesmo é reprisentado por duas espécies descritas sob o gênero Stipa, S. panicoides Lam. e S. bicolor Vahl, enquanto que em Doell (1878), o gênero é representad`s por várias espécies descritas sob os gêneros Urachne e Stipa.

Dados sobre espécies oc orrentes no Brasil podem ser encontrados em

1 - Curso de Pós-Graduação em Botânica, UFRGS.

2 - Depto de Botânica UFRGS - . Av. Paulo Gama, s/no 90049

Porto Alegre, RS - Brasil. B olsista de Pesquisa CNPq. 
floras de regiōes limítrofes, como Burkart (1969), Cabrera (1970), Correa (1978) e Rosengurtt et al. (1970). Especificamente para o Brasil, não existe nenhum trabalho sobre o gênero, sendo o mesmo incluído em floras de regiōes limitadas. Assim, Araújo (1971) cita 8 espécies para o Rio Grande do Sul, Smith et al (1982) 6 para Santa Catarina, e Renvoize (1988) cita 2 para o $\mathrm{Pa}$ raná.

As espécies de Piptochaetium são microtérmicas, de ciclo hibernal e, como parte dos campos naturais do sul do Brasil, contribuem para a manutenção do gado na época crítica do inverno. Os antécios de algumas espécies conhecidas por "flechilhas", devido ao seu calo agudo e pungente, são citados como prejudiciais por ferir a pele do gado e prender-se à lã dos ovinos (Araújo, 1971; Rosengurtt et al., 1970).

Este trabalho visa fornecer subsídios para a identificação das espécies ocorrentes no Brasil, como uma contribuição ao conhecimento fitossociológico e ecológico dos campos naturais.

\section{Material e métodos}

Os resultados apresentados foram obtidos a partir da revisão bibliográfica, coleta e observação das populações no campo e revisão dos seguintes herbários:

BLA - Herbário do Instituto de Pesquisas Zootécnicas "Francisco Osório", Secretaria da Agricultura do Estado do Rio Grande do Sul, Porto Alegre, RS, Brasil.

CTES - Herbário del Departamento de Botánica y Ecología, de la Facultád de Agronomia y Veterinaria, Universidad del Nordeste, Corrientes, Argentina.

GUA - Herbário Alberto Castelhanos, Fundação Estadual de Engenharia do Meio Ambiente, Rio de Janeiro, RJ, Brasil.

HAS - Herbário "Prof. Dr. Alarich Schultz”, Museu de Ciências Naturais da Fundação Zoobotânica do Rio Grande do Sul, Porto Alegre, RS, Brasil.

HBR - Herbário Barbosa Rodrigues, Itajaí, SC, Brasil.

ICN - Herbário do Departamento de Botânica do Instituto de Biociências da Universidade Federal do Rio Grande do Sul, Porto Alegre, RS, Brasil.

MVFA - Herbário del Laboratorio de Botánica, Facultad de Agronomia, Montevideo, Uruguay.

PACA - Herbário Anchieta, Universidade Vale do Rio dos Sinos, São Leopoldo, RS, Brasil.

PEL - Herbário do Departamento de Botànica, do Instituto de Biologia da Universidade Federal de Pelotas, RS, Brasil.

PKDC - Herbário Per Karl Dusén, Curitiba, Paraná, Brasil. 
R - Herbário do Museu Nacional do Rio de Janeiro, RJ, Brasil.

RB - Herbário do Jardim Botânico do Rio de Janeiro, RJ, Brasil.

SMDB - Herbário do Departamento de Biologia, Centro de Ciências Naturais e Exatas, Universidade Federal de Santa Maria, RS, Brasil.

SP - Herbário do Estado "Maria Eneyda P.K. Fidalgo", Instituto de Botânica, São Paulo, SP, Brasil.

Devido ao objetivo principal do trabalho ser o fornecimento de meios práticos para a identificação das espécies, são citados no material examinado apenas parte dos exemplares, procurando englobar toda a área de distribuição de cada espécie dentro do Brasil. Uma listagem completa do material examinado pode ser solicitada ao segundo autor.

O material obtido nas coletas realizadas encontra-se depositado no herbário do Departamento de Botânica da Universidade Federal do Rio Grande do Sul (ICN).

Os dados de distribuição apresentados são baseados no material examinado e na literatura citada no item "Introdução" deste trabalho, além de Parodi (1944) e Thomasson (1980).

\section{Resultados e conclusões}

Foi confirmada, até o presente, a ocorrência de 14 entidades taxonômicas, 8 espécies e 6 variedades, para o Brasil.

A área de maior concentração de espécies no Brasil está no Rio Grande do Sul, diminuindo em direção norte, sendo que o limite setentrional do gênero se encontra aproximadamente na latitude do Trópico de Capricórnio, onde chega uma só espécie, $P$. montevidense.

\section{Caracterızaçao do genero}

Piptochaetium Presl, Rel. Haenkeanae, 1:222.1830.

Caryochloa Spreng., Syst. Veget. Curs. Post. 4(2): 22 e 30.1827.

Urachne, Sect. 5 Piptochaetium (Presl) Trin. \& Rupr. Acta Acad. Petrop. 6,5: 22. 1842.

Oryzopsis Speg., An. Mus. Nac. Montevideo. 4 (2): 1.1901.

Plantas perenes, cespitosas. Bainhas foliares abertas. Lâminas foliares planas, convolutas ou conduplicadas, glabras ou pilosas. Lígulas membranosas.

Inflorescências em panículas laxas, subcontraídas ou contraídas. Espìguetas unifloras. Glumas lanceolado-acuminadas, persistentes na inflorescência, geralmente mais longas que o antécio. Lemas rígidos, oblanceolados, 
obovados ou largamente obovados, castanho ou castanho-escuro, com o ápice formando uma coroa larga ou estreita, reduzida ou engrossada, na qual se insere a arista, esta persistente ou caduca; base diferenciada em um calo agudo ou obtuso, glabro ou piloso; margens involutas sobre a pálea; superfície glabra ou pilosa, somente estriada longitudinalmente, papilosa ou verrucosa. Pálea rígida, navicular, bicarenada, sulcada longitudinalmente. Lodículas 3, lanceoladas. Estames 3, anteras freqüentemente pilosas no ápice. Cariopses com sulco ventral longitudinal, hilo linear.

Espécie-tipo: Piptochaetium setifolium Presl

Chave preliminar para as espécies de Piptochaetium Presl do Brasil

A. Lema piloso em toda a extensão

B. Glumas maiores que o lema. Pêlos leonados, com ca. de 3,0mm, ocultando totalmente o lema e sobrepassando amplamente a coróa. Lema com $(2,6)-3,0-3,5-(4,0) \mathrm{mm}$ de comp. (Fig. 1A) ...P. lasianthum

BB. Glumas inferiores menores que o lema, as superiores maiores. Pêlos castanho-claros, com ca. de $0,5 \mathrm{~mm}$, não ocultando o lema e não sobrepassando a coroa. Lema com $(4,2)-4,5-5,0 \mathrm{~mm}$ de comp. (Fig. 1B). . . . . alpinum

AA. Lema glabro, apenas o calo piloso.

C. Calo agudo, pungente, com 1,4 - 3,5-(4,6)mm de comp. Glumas com $6,4-17,0-(18,0) \mathrm{mm}$ de comp. Lema com arista persistente, com 23 - 75 - (90)mm de comp. Lema obovado ou oblanceolado, com 3,9-10,0-(12,0)mm de comp.

D. Arista com 23 - 40 - (45)mm de comp.; calo com 1,4$2,0 \mathrm{~mm}$ de comp. Lema obovada com $3,9-5,5-(7,0) \mathrm{mm}$ de comp. Glumas com 6,4-11,0mm de comp.

E. Lema com 3,9 - 4,5mm de comp.; arista com $23-$ $31 \mathrm{~mm}$ de comp.; calo com 1,4-1,5mm de comp. Glumas com $6,4-7,8 \mathrm{~mm}$ de comp. (Fig. 1C) . . . . . . 
EE. Lema com 4,8 - 5,5 - (7,0)mm de comp.; arista com $35-40-(45) \mathrm{mm}$ de comp.; calo com 1,4-2,0mm de comp. Glumas com 7,5-11,0mm de

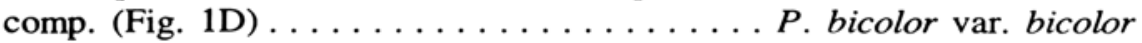

DD. Arista com $50-75-(90) m m$ de comp.; calo com $(2,2)-2,5-3,5$ - (4,6)mm de comp. Lema oblanceolado, com $(6,2)-7,0-10,0-$ $(12,0) \mathrm{mm}$ de comp. Glumas com 12,0 - 17,0 - $(18,0) \mathrm{mm}$ de comp.

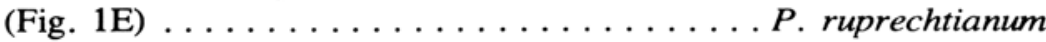

CC. Calo obtuso, não pungente com 0,2-0,4mm de comp. Glumas com $2,2-7,8-(8,0) \mathrm{mm}$ de comp. Lema com arista caduca ou destacável sob press ão, com $5-28 \mathrm{~mm}$ de comp. Lema desde muito largamente obovado até obovado ou elíptico, $\operatorname{com}(1,2)$ $-1,4-4,2 \mathrm{~mm}$ de comp.

F. Arista com $5-8-(10) m m$ de comp., caduca Lema $\operatorname{com}(1,2)-1,4-$ $1,8-(2,0) \mathrm{mm}$ de comp., muito largamente obovado; calo com ca. de $0,2 \mathrm{~mm}$ de comp.

G. Lema estriado longitudinalmente, com ou sem escassa papilas próximas da coroa.

H. Lema sem papilas (Fig. 1F) ... P. panicoides var. panicoides

HH. Lema com escassas papilas próximas da coroa (Fig. 1G) ... P. panicoides var. subpapillosum

GG. Lema verrucoso em toda a extensão. (Fig. 1H) $P$. montevidense

FF. Arista com $14-28 \mathrm{~mm}$ de comp., destacável sob pressão. Lema com $(2,1)-2,2-4,2 \mathrm{~mm}$ de comp., desde muito largamente obovado até obovado ou elíptico; calo com $0,3-0,4 \mathrm{~mm}$ de comp.

I. Coroa com 0,3-0,4mm de diâm., de margem não engrossada. Lema com $(2,1)-2,2-3,0-(3,3) \mathrm{mm}$ de comp., totalmente verrucoso; arista com (15) $-18-20-(25) \mathrm{mm}$ de comp.

J. Lema obovado com $2,4-3,0-(3,3)$ mm de comp., $1,0-$ $1,2 \mathrm{~mm}$ de larg. (Fig. 1I) . P. uruguense var. uruguense

JJ. Lema largamente obovado com 2,1-2,2mm de comp., $1,3-1,4 \mathrm{~mm}$ de larg. (Fig. $1 \mathrm{~J}) P$. uruguense var. microcarpum 
II. Coroa com 0,7 - 1,7mm de diâm., de margem engrossada. Lema com 2,5$4,2 \mathrm{~mm}$ de comp., com ou sem verrugas; arista com $14-28 \mathrm{~mm}$ de comp.

L. Coroa com 1,3-1,7mm de diâm. e 0,2 - 0,5mm de altura. Lema largamente obovado.

M. Lema com verrugas pronunciadas (ca. $2,0 \mathrm{~mm}$ de altura) em toda a superfície, com 2,3-2,6mm de comp., 1,8 - 1,9mm de larg. Coroa com $0,35-0,5 \mathrm{~mm}$ de altura. Lâminas foliares pouco pilosas. (Fig. 1L) . . . . . . P. stipoides var. echinulatum

MM. Lema com verrugas pouco pronunciadas (menores de $0,1 \mathrm{~mm}$ de altura) no $1 / 3$ superior, $\operatorname{com}(2,5)-2,6-3,4 \mathrm{~mm}$ de comp., $1,9-2,4-(2,6) \mathrm{mm}$ de larg. Coroa com $0,2-$ $(0,3) \mathrm{mm}$ de altura. Lâminas foliares densamente pilosas. (Fig. 1M). . . . . . . . P. stipoides var. chaetophorum

LL. Coroa com 0,7-0,9mm de diâm. e 0,2-(0,3)mm de altura. Lema muito largamente obovado ou elíptico.

N. Lema elíptico, com verrugas escassas e diminutas perto da coroa, com $(3,2)-3,4-4,2 \mathrm{~mm}$ de comp., $1,7-1,8-$ $(2,0) \mathrm{mm}$ de larg. (Fig. 1N) . . .P. stipoides var. stipoides

NN. Lema muito largamente obovado, sem verrugas ou com escassas e diminutas perto da coroa, com 2,5 - 2,9 $(3,1) \mathrm{mm}$ de comp., $(1,6)-1,8-2,0-2,0 \mathrm{~mm}$ de larg. (Fig. 1O) . . . . . . stipoides var. purpurascens 


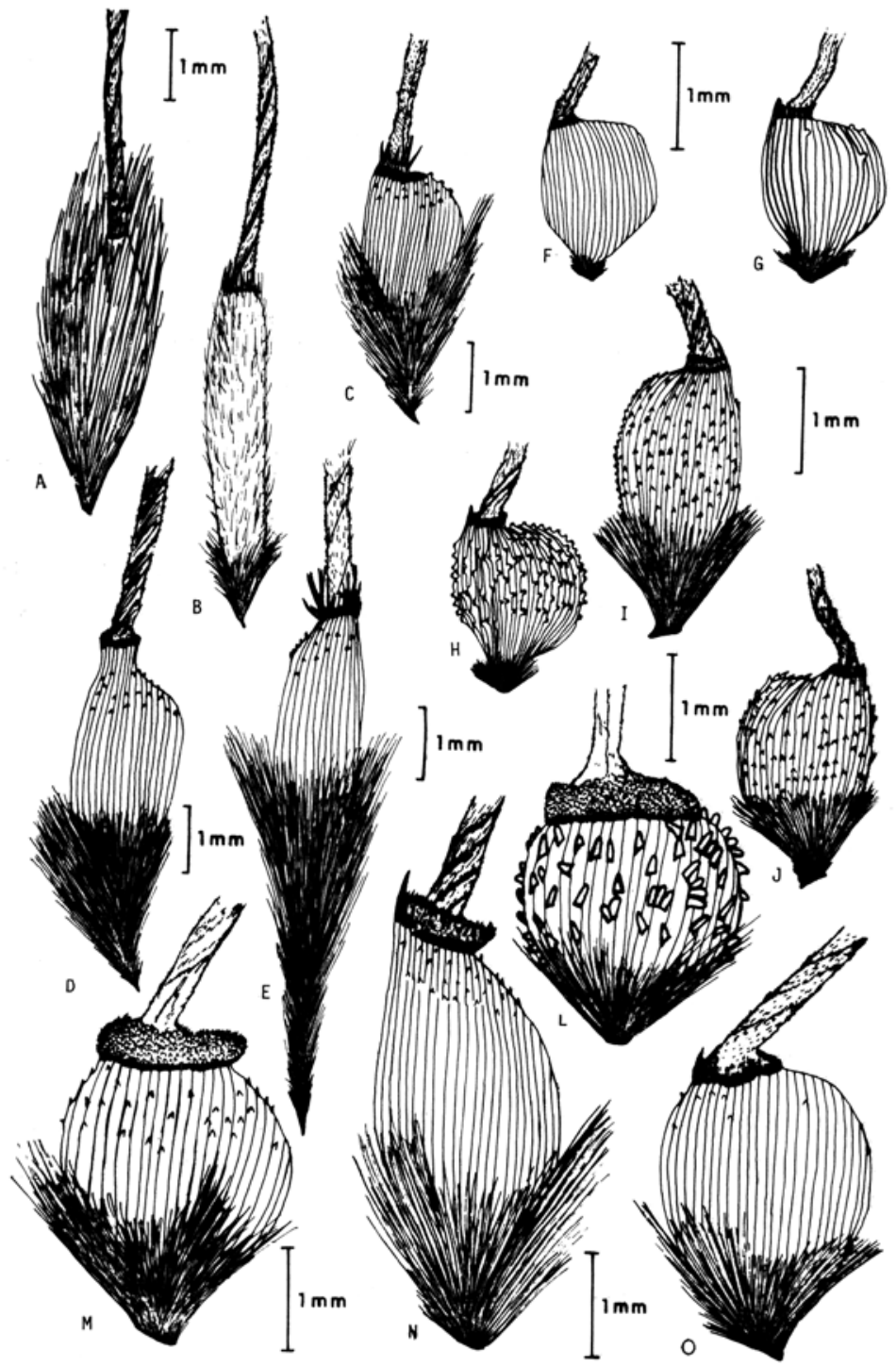

Figura 1 - Antécios com aristas parcialmente removidas de: A: $P$. lasianthum (J. Mujica 81 - ICN); B: P. alpinum (A. Zanin e: (tl. - ICN); C: P. bicolor var. menor (M. Marchi 53 - ICN); D: P. bicolor var. bicolor (Pott 84 - BLA); E: P. ruprechtianum (J. Mujica 36 - ICN); F: P. panicoides var. pcricoides (J. Mujica 74A - ICN); G: P. panicoides var. subpapillosum (J. Mujica 46 - ICN); H: $P$. montevidense (J. Mujica $39-\mathrm{ICN}) ; \mathrm{I}:$ P. uruguense var. microcarpum $(\mathrm{P}$. Brack - ICN); J: P. uruguense var. microcarpum (P. Brack ICN); L: P. Atipoides var. Echinatum (Swallen 7669 - PEL); M: P. stipoides var. chaetophorum (J. Mujica 63 - ICN); N: P. stipoides var. stipoides (Simas - BLA 1362); O: P. stipoides var. purpurascens (Simas - BLA 6642). 
A - Piptochaetium lasianthum Griseb. Symb. Flor. Arg, 297. 1879. (Fig. 1)

Distribuição geográfica: Argentina, Brasil e Uruguai. Brasil: Rio Grande do Sul e Santa Catarina.

Prefere solos rochosos, formando agrupamentos vistosos devido aos pêlos leonados dos lemas.

Esta espécie e $P$. alpinum Smith são as únicas espécies ocorrentes no Brasil que apresentam lemas pilosos em toda a extensão.

Material examinado: Rio Grande do Sul: Bom Jesus, em direção a Vacaria, 28/XI/1988, J. Mujica 113 (ICN); Porto Alegre, Morro da Polícia, 14/XI/1988, J. Mujica 80 (ICN); Santa Vitória do Palmar, 17/XI/1945, Swallen (PEL 10171). Santa Catarina: Água Doce, Campo de Palmas, 34/XII/1964, Smith \& Klein 13418 (HBR); Bom Jardim da Serra, 15/XI/1958, Reitz \& Klein 7927 (HBR).

B - P. alpinum Smith, Phytologia 22: 89, tab. 1. figs. 14, 15, 1971.

Ocorrência restrita ao Brasil, nos campos da região dos Aparados da Serra Geral, no Rio Grande do Sul e Santa Catarina, em altitudes de 1000 a 1400 metros.

Apresenta as glumas inferiores menores do que o antécio, o que a diferencia das demais espécies do gênero aqui tratadas, nas quais as duas glumas sobrepassam o antécio.

Material examinado: Rio Grande do Sul: Cambará do Sul, Itaimbezinho, 3/XII/1971, Valls et al 1875 (MVFA). Santa Catarina: Bom Jardim da Serra, 10/XII/1958, Reitz \& Klein 7710 (HBR).

C - P. bicolor (Vahl) Desv. var. minor (Speg.) Parodi, Rev. Mus. La Plata Secc. Bot. 6: 256. 1944.

Oryzopsis bicolor (Vahl) Speg. var. minor Speg An. Mus. Nac. Montevideo (4) 2: 8.1901.

Distribuição geográfica: Argentina, Chile, (Rosengurtt et al., 1970), Brasil e Uruguai. Brasil: Rio Grande do Sul. Até o momento só representada por uma coleta.

Material examinado: Rio Grande do Sul: Bagé, entrada da Embrapa, 11/11/1989, M. Marchi 53 (ICN). 
D - P. bicolor (Vahl) desv. var. bicolor, in Gay, Flor. Chil. 6: 273. 1853. Stipa bicolor Vahl, Symb. Bot. 2: 24. 1791.

Material examinado: Rio Grande do Sul: Guaíba, Estação Experimental Agronômica, 11/XI/1988, J. Mujica $7 O$ (ICN); Pelotas, I.A.S., 17/XI/1954, Sacco 239 (RB); Santana do Livramento, 16/XII/1955, Barreto (BLA 1351).

E - P. ruprechtianum Dissv. in Gay, Flor. Chil. 6: 274. 1853.

Stipa bicolor Trin. \& Rupr. Act. Acad. Petrop. 6,5: 26. 1842.

P. confusum Parodi, Rev. Mus. La Plata, Secc. Bot. 6: 248. 1944.

Syn. nov.

Distribuição geográfica: Argentina (Parodi, 1944), Brasil e Uruguai. Brasil: Rio Grande do Sul, Santa Catarina e Paraná.

Ocorre em diversos tipos de solos, sendo bastante freqüente.

Material examinado: Rio Grande do Sul: Bom Jesus, 6/I/1988, A. Zanin 80 (ICN); Guaíba, Estação Experimental Agronômica, 28/X/1988, J. Mujica 24 (ICN); São Borja, $52 \mathrm{~km}$ em direção a Santiago, 20/XII/1972, Norman et al. (BLA 7930). Santa Catarina: Lages, 1/I/1946, Swallen 8054 (PEL). Paraná: Palmas, Sete Butieiros, Hatschbach 30757 (SP).

F - P. panicoides (Lam.) Desv. var. panicoides, in Gay. Flor Chil. 6:270, lám. 75, f. 2. 1853.

Stipa panicoides Lam. Illust. gen. 1: 158. 1791.

Distribuição geográfica: Argentina, Chile, Colômbia (Rosengurtt et al. 1970), Bolívia e Peru (Cabrera, 1970), Brasil, Uruguai e Venezuela.

Esta variedade e a variedade subpapillosum ocorrem no Rio Grande do Sul, na região litorânea, em solos arenosos.

Material examinado: Rio Grande do Sul: Guaíba, Estação Experimental Agronômica, 11/XI/1988; J. Mujica 60 (ICN); Pelotas, IPEAS-UFPEL, 27/IX/1972, Luz et al.39 (ICN); Porto Alegre, Morro Santana, 4/XI/1988, J. Mujica 44 (ICN).

G-P. panicoides (Lam.) Desv. vasr. subpapillosum (Hack). Petetin, in Correa, Flor. Patag. 3: 339. 1969.

P. leiocarpum (Speg.) Hack. f. subpapillosa Hack., in Stuckert, An. Mus. Nac. Bs. Aires 13: 463.1906. 
Distribuição geográfica: Argentina, Bolívia (Correa, 1978), Brasil, Peru e Uruguai.

Material examinado: Rio Grande do Sul: Porto Alegre, Morro Santana, 4/XI/1988, J. Mujica 46 (ICN).

H - P. montevidense (Spreng.) Parodi, Rev. Fac. Agr. y Vet. B. Aires 7:163. 1930.

Caryochloa montevidensis Spreng. Syst. veg. 4 (2): 30. 1827.

Distribuição geográfica: Bolívia (Parodi, 1944), Argentina, Brasil, Chile, Paraguai e Uruguai. Brasil: Rio Grande do Sul, Santa Catarina, Paraná e São Paulo.

Entre as espécies ocorrentes no Brasil é a mais comum nos campos e a que apresenta distribuição mais ampla.

Material examinado: Rio Grande do Sul: Guaíba, Estação Experimental Agronômica, 28/X/1988, J. Mujica 39 (ICN); Santana do Livramento, Estação Experimental, 1/XI/1974, A. Castro (BLA 11739); São José dos Ausentes para Bom Jesus, 28/XI/1988, J. Mujica 118 (ICN). Santa Catarina: Água Doce, 6/XII/1971, Smith et al. 15698 (HBR); Campo Alegre, 19/X/1957, Reitz \& Klein 5334 (ICN). Paraná: Curitiba, 30/XI/1903, Dusén 2381 (R). São Paulo: Campos do Jordão, 4/I/1981, Werner (SP 169259).

I - P. uruguense Griseb. var. uruguense, Symb. Flor. Arg. 297.1879.

Distribuição geográfica: Argentina, Brasil e Uruguai. Brasil: Rio Grande do Sul e Santa Catarina, pouco freqüente.

Material examinado: Rio Grande do sul: Bagé, 1/XI/1945, Swallen 7561 (PEL); Esmeralda, Estação Ecológica de Aracurí, 29/XI/1988, J. Mujica 143 (ICN); Santo Ângelo, 16/XI/1974, Silas 79 (ICN): Santa Catarina: Água Doce, 23/XII/1956, Smith \& Reitz 9124 (HBR).

J - P. uruguense Griseb. var. microcarpum Parodi, Rev. Mus. La Plata Secc. Bot. 6:290. 1944.

Distribuição geográfica: Paraguai (Parodi, 1944), Argentina, Brasil e Uruguai. Brasil: Rio Grande do Sul, pouco freqüente.

Material examinado: Rio Grande do Sul: São Borja, para Santiago, 20/XII/1972, Boldrini et al. (BLA 7876); Vacaria, 9/XI/1946, Swallen 8213 
(PEL).

L - P. stipoides (Trin. \& Rupr.) Hack. var. echinulatum Parodi, Rev. Mus. La Plata Secc. Bot. 6:271. 1944.

Distribuição geográfica: Argentina, Chile, (Rosengurtt et al., 1970), Brasil e Uruguai. Brasil: Rio Grande do Sul.

Até o momento só foram encontrados 2 exemplares.

Material examinado: Rio Grande do Sul: Alegrete, Guarita, 26/XII/1958, J. Mattos 7094 (BLA); Uruguaiana, Posto Zootécnico, 9/XII/1945, Swallen 7669 (PEL).

M - P. stipoides (Trin. \& Rupr.) Hack. var. chaetophorum (Griseb.) Parodi, Rev. Mus La Plata Secc. Bot. 6: 267. 1944.

Piptochaetium chaetophorum Griseb. Symb. Flor. Arg. 298. 1879.

Distribuição geográfica: Chile (Parodi, 1944), Argentina, Brasil e Uruguai. Brasil: Rio Grande do Sul e Santa Catarina.

É a variedade de $P$. stipoides mais freqüente no Brasil, sendo caracterizada vegetativamente por suas lâminas foliares densamente pilosas.

É comum encontrar antécios imaturos desta variedade que apresentam bastante semelhança com a variedade stipoides. Porém, no mesmo exemplar encontra-se geralmente antécios maduros de forma típica, mostrando uma associação entre a forma e o estágio de desenvolvimento dos mesmos.

Material examinado: Rio Grande do Sul: Bagé, 28/XI/1945, Swallen 7466 (PEL); Bom Jesus, XII/1954, Barreto (BLA 1119); Porto Alegre, 14/XII/1988, J. Mujica 86 (ICN). Santa Catarina: Lages, 5/1/1946, Swallen 8156 (PEL).

N - P. stipoides (Trin. \& Rupr.) Hackivar. stipoides, in Arechavaleta, An. Mus. Nac. Montevideo 1(4): 328. 1896.

Urachne stipoides Trin. \& Rupr. Act. Acad. Petrop. 6, 5: 25.1842.

Distribuição geográfica: Chile, Colômbia, Uruguai (Rosengurtt et al , 1970), México (Thomasson, 1980), Argentina e Brasil. Brasil: Rio Grande do Sul, pouco freqüente.

Foi confundida por Araújo (1971) com Piptochaetium setosum (Trin.) Arech., a qual não ocorre no Brasil. 
Material examinado: Rio Grande do Sul: Bagé, 2/XII/1945, Swallen 7569 (PEL); Guaíba, Instituto de Plantas Forrageiras, 19/XI/1965, Barreto (BLA 2220); Santana do Livramento, XI/1936, E. Xavier (BLA 5350):

O - P. stipoides var. purpurascens (Hack.) Parodi, Rev. Mus. La Plata Secc. Bot. 6: 272. 1944.

Piptochaetium ovatum Desv. var. purpurascens Hack. in Stuckert, An. Mus. Nac. B. Aires. 21: 86. 1911.

Distribuição geográfica: Argentina, Brasil e Uruguai. Brasil: Rio Grande do Sul, comum.

Material examinado: Rio Grande do Sul: Alegrete, Porto Velho, 10/XII/1955, Barreto (BLA 1308); Rosário do Sul, 8/XII/1971, Jung 59 (ICN); Santa Vitória do Palmar, 17/XI/1945, Swallen 7392 (PEL).

Até o momento foram encontradas 14 entidades taxonômicas de Piptochaetium para o Brasil, 13 das quais ocorrem também na Argentina e Uruguai.

Sua maior concentração ocorre no Rio Grande do Sul, 6 espécies chegando até Santa Catarina, 2 no Paraná e apenas $P$. montevidense alcançando a região de São Paulo, limite norte de distribuição no Brasil até agora conhecido para o gênero.

$P$. alpinum é exclusivo do Brasil, com ocorrência restrita aos campos dos Aparados da Serra Geral do Rio Grande do Sul e Santa Catarina.

É proposta a sinonimização de $P$. confusum Parodi sob $P$. ruprechtianum Desv.

\section{Referências bibliográficas}

ARAÚJO, A. A. 1971. Principais gramíneas do Rio Grande do Sul. Porto Alegre, sulina.

BURKART, A. 1969. Flora Ilustrada de Entre Rios (Argentina) Gramíneas. Buenos Aires, INTA.

CABRERA, A. L. 1970. Flora de la Provincia de Buenos Aires. Buenos Aires, INTA.

CORREA, M. N. 1978. Flora Patagónica. Buenos Aires, INTA.

DOELL, J. C. 1878. Gramineae. In: C.F.P. MARTIUS. Flora Brasiliensis. Monachii, Fleischer.

NEES, C.G. 1829. Agrostologia Brasiliensis. Stuttgartie et Tubingae, J. G. Cottae. v.2.

PARODI, L. R. 1944. Revisión de las Gramíneas australes Americanas del Género Piptochaetium. Rev. Museo La Plata Secc. Bot. 6: 213-310.

RENVOIZE, S. A. 1988. Hatschbach's Paraná Grasses. Kew, Royal Botanic 
Gardens.

ROSENGURTT, S. B.; B. ARRILLAGA DE MAFFEI; P. IZAGUIRRE DE ARTUCIO, 1970. Gramineas Uruguayas. Montevideo, Univ. Publ.

SMITH, L. B.; D.C. WASSHAUSEN \& R. M. KLEIN. 1982. Gramíneas. Flora Ilustrada Catarinense. Itajaí.

THOMASSON, J.R. 1980. Distribution in Piptochaetium (Gramineae - Stipeae): Paleophytogeographical. Significante. Iselya 1(4): 161-165. 